\title{
Multi-Fidelity Design of Low-Thrust Resonant Captures for Near-Earth Asteroids
}

\author{
Rita Neves* and Joan-Pau Sánchez ${ }^{\dagger}$ \\ Cranfield University, College Road, MK43 OAL, Cranfield, UK
}

\begin{abstract}
The design of a space trajectory is strongly linked to the gravitational and non-gravitational environment and the dynamical frameworks required to model it. These dynamical models may range from low to high-fidelity, with corresponding computational costs. This paper proposes a multi-fidelity approach for the computation of nearly resonant trajectories with the Earth. This framework is used to compute trajectories for the capture of Near-Earth Asteroids (NEA) into Libration Point Orbits (LPO) of the Sun-Earth system. The transfer is first computed in a suitable low-fidelity model—the Keplerian Map—and a multi-fidelity approach is subsequently used to refine the solution from an impulsive approximation into a low-thrust transfer in the Circular Restricted Three-Body Problem. The entire trajectory follows a nearly resonant motion with the Earth, lasting less than two synodic periods: starting when the retrieval spacecraft attaches itself to the asteroid, they will encounter the Earth twice, being captured into the target orbit at the end of the second encounter. A velocity change maneuver is carried out at the beginning of the motion, so that the first encounter with the Earth provides a gravitational perturbation resulting on a reduction of overall propellant costs of the transfer. The developed framework is very flexible in terms of the desired accuracy and allows for the low computational cost exploration of a vast number of possible trajectories. The obtained low-thrust transfers yield, for six asteroids, a much higher retrievable mass in comparison with direct capture trajectories, which do not undertake Earth-resonant encounters.
\end{abstract}

\section{Introduction}

Our Solar System is swarmed by millions of minor bodies, including asteroids and comets. These minor celestial bodies have received significant attention in recent years, since their study may hold the key to understand the formation and evolution of our Solar System. NASA, ESA, JAXA and recently also the CNSA (China National Space Administration) have conceived and launched a series of missions to obtain data from such bodies; particularly, from Near-Earth Objects (NEOs). NEOs have come under the spotlight because of two important aspects: they are among

\footnotetext{
*PhD Candidate, School of Aerospace, Transport and Manufacturing, Centre for Cyberphysical Systems, r.neves@cranfield.ac.uk, Student AIAA Member

${ }^{\dagger}$ Associate Professor, School of Aerospace, Transport and Manufacturing, Centre for Cyberphysical Systems, jp.sanchez@cranfield.ac.uk
} 
the easiest celestial bodies to reach from the Earth and may also represent a threat to our planet [1].

Furthermore, the concept of utilization of in-space resources is also receiving a renewed attention. This would open the possibility for otherwise unaffordable mission scenarios to start being pursued [2]. More scientists and engineers are now advocating for the necessary technologies to prospect, mine and utilize materials in space [3]. Asteroids are again particularly appealing sources of potential resources, since they contain a plethora of useful materials [4].

Amid these trends, asteroid retrieval, or capture, missions were proposed. The concept envisages a spacecraft that performs a rendezvous with an asteroid, lassos it and then hauls it back to the Earth's neighborhood, so that it can be more easily accessed. The mission has clear synergies with all three of the above aspects of asteroid missions; science, planetary defense and mining.

The concept of retrieving asteroids was first conceived by rocketry futurists [5, 6], early in the space age, although its original root may likely be traced back into classical science fiction. However, a much more level-headed concept, together with a serious effort to develop it, have been pursued very recently within NASA's Asteroid Initiative [7].

The Asteroid Initiative was announced in 2013 and, among other activities, it included the Asteroid Redirect Robotic Mission (ARRM), aimed at hauling an asteroid to a Lunar Distant Retrograde Orbit (LDRO). The original ARRM concept was proposed in 2011 after a feasibility study workshop at the Keck Institute of Space Studies [8]. Since then, several developmental activities have advanced key technologies for the mission, such as high power solar electric propulsion and the asteroid capture inter-phase mechanism. Nevertheless, these activities have been put on hold by the current US administration [9].

The focus of this paper is on the design of capture trajectories for asteroid retrieval missions. Many asteroids have masses several orders of magnitude larger than that of the typical interplanetary spacecraft $\left(\sim 10^{3} \mathrm{~kg}\right)$. As such, for a capture to be feasible, it may have to be done using extremely low-energy transfers. These are pathways that exploit the simultaneous gravitational interactions from the Sun, planets and moons [10]. NASA's ARRM trajectories, for example, aimed at inserting the asteroid (or part of it) into a LDRO [11, 12]. In order to accomplish such a trajectory, the gravitational interactions of the Sun, Earth and Moon needed to be considered very carefully.

Several endgame orbits for captured asteroids have been studied in literature, each implying different methodologies to approach the design of the capture transfer. A substantial amount of work has not targeted a specific final capture orbit, but has instead considered the energy conditions to enable permanent (or quasi-permanent) capture within the Earth's sphere of influence [13-18]. Another common approach is to target a specific final orbit for the captured object: LDROs for NASA's ARRM concept [11, 12], Sun-Earth Libration Point orbits (LPOs) [19-22] or Earth-Moon LPOs [23, 24].

The exploitation of encounters with other bodies in the Solar System for asteroid capture has been previously studied, particularly swing-bys of the Moon [12, 17] and Earth [18], using the patched conic approximation. However, the most Easily Retrievable Objects [21] of the NEA population move on such a regime of motion that they rarely get close enough to the Earth for a swing-by maneuver. Nevertheless, even if the asteroid undergoes an encounter with the 
Earth outside its sphere of influence, its gravitational interaction will still be noticeable and may substantially modify the asteroid's trajectory. Thus, the current paper focuses on the design of retrieval trajectories to transfer asteroids into Sun-Earth LPOs. However, this work goes beyond previous publications [21, 22, 25] by including multiple Earth encounters along the capture trajectory, occurring outside the sphere of influence of the Earth in a low-energy regime, and solving the optimal control problem for the final low-thrust transfer.

For this work, the patched conic approximation is not a suitable dynamical model to estimate the Earth's perturbative effects during an encounter. Even within the Earth's sphere of influence, this model does not render a good accuracy in a low-energy regime [26], which is relevant for the type of asteroid that requires a minimum energy to be captured [27, 28]. Nevertheless, the approach of using a lower-fidelity model, as long as it captures well the underlying dynamics that we seek to control, is still a reasonable approach to explore the infinite continuum of possible trajectories. Thus, this paper proposes a novel low-fidelity model which is based on semi-analytical approximation of the Circular Restricted Three-Body Problem (CR3BP): the Keplerian Map (KM). A multi-fidelity approach is then used to refine the solution from an impulsive approximation, in a low-fidelity dynamical model, into a low-thrust transfer in the CR3BP.

The paper is organized in the following way: Section II provides an overview of the different dynamical frameworks used. Section III] defines the impulsive approximation capture trajectory in depth, as well as the design process. Section IV describes the low-thrust optimal control problem, and the process of solving it from the initial impulsive seeds obtained from Section [II] Finally, Section $\mathrm{V}$ showcases and discusses the results obtained, while Section $\mathrm{VI}$ highlights the conclusions of the work.

\section{Multi-Fidelity Dynamical Framework}

In order to choose the dynamical model in which to compute the motion of a body in space, a trade-off has to be done between accuracy and computational cost. Besides, the chosen one has to take into consideration the perturbative accelerations caused by nearby celestial bodies.

Instead of choosing only one model, this paper proposes a multi-fidelity framework for the asteroidfotmotion in a capture mission scenario. In this way, a sequential pruning of the NEA population can be made with increasingly higher fidelity models. Thus, with adequate accuracy, it is possible to compute an extensive number of trajectories with lower computational cost, and refine only the interesting ones.

As such, the asteroid's trajectory is first computed with a lower-fidelity model—the KM-and then refined into a CR3BP motion. Both models are described separately in this section and their accuracy and computational costs are compared later on, in Sub-section V.D The framework leaves the possibility of further refinement beyond the CR3BP, using for instance homotopy continuation methods [29]; this is, however, out of the scope of this paper.

\footnotetext{
*For the sake of simplicity, the system formed by the coupling of the spacecraft with the asteroid will be henceforth referred to as the asteroid.
} 


\section{A. Circular Restricted Three-Body Problem}

In its general form, the motion of any system with more than two bodies is not integrable, which means no analytical solution exists. The motion of a dynamical system composed of three bodies can be computed numerically; for that purpose, the CR3BP is used. This framework appears as a simplification on the three-body problem (3BP), in which the third body's mass is deemed insignificant compared to the other two, the primaries, and their orbits are circular around each other.

The CR3BP is typically represented in the synodic reference frame; all the physical quantities are normalized in such a way that both the sum of the primaries' masses and the distance that separates them is equal to 1 [30]. The normalized position and velocity in this model obey the following equations of motion:

$$
\begin{aligned}
\ddot{x}-2 \dot{y}-x & =-\frac{(1-\mu)(x+\mu)}{r_{1}{ }^{3}}-\frac{\mu(x-1+\mu)}{r_{2}{ }^{3}} \\
\ddot{y}+2 \dot{x}-y & =-\frac{(1-\mu) y}{r_{1}{ }^{3}}-\frac{\mu y}{r_{2}{ }^{3}} \\
\ddot{z} & =-\frac{(1-\mu) z}{r_{1}{ }^{3}}-\frac{\mu z}{r_{2}{ }^{3}}
\end{aligned}
$$

where $r_{1}$ and $r_{2}$ are the distances to each of the primaries and $\mu$ is the normalized gravitational parameter of the system.

Since these equations can only be solved numerically and the influence of the two primaries is computed throughout the entirety of the third body's motion, this is a higher fidelity model than the KM, bearing greater computational cost. This claim will be substantiated in Sub-section V.D

\section{LPOs and Invariant Manifolds}

In the synodic reference frame of the CR3BP, there are five equilibrium points in which a third body can maintain the same position relative to the primaries. These are called the libration points, caused by the gravitational interactions in the CR3BP.

Several types of periodic orbits can be found around them, from which the following can be highlighted: Horizontal Lyapunov orbits, in the orbital plane of the motion of the secondary; Vertical Lyapunov orbits, horizontally symmetric and shaped like a figure-eight; Halo orbits, which bifurcate from the Horizontal Lyapunov orbit family. These orbits are regarded as interesting targets for asteroid retrieval, as they are located in convenient positions in regards to the Solar System and require little station-keeping [31].

Connected to the LPOs, one can find invariant manifolds—dynamical structures composed of countless orbits [32]. In this way, the goal of moving the asteroid into an LPO requires a trajectory design that inserts the asteroid into one of its invariant manifold orbits; then, needing nearly no further maneuvering, the asteroid will reach the desired target. 


\section{B. The Keplerian Map}

When computing the motion of an object in the Solar System, the simplest dynamical model assumes that there is only one body exerting gravitational influence on it. This is the case of the previously mentioned two-body problem, whose equations of motion can be analytically solved.

The KM is a general perturbation technique first formulated by Ross and Scheeres [33] and then expanded into three-dimensional motion by Alessi and Sánchez [34]. The KM accounts for the disturbing acceleration of a third-body in two-body motion. Its use is very fitting for the study of Earth-resonant encounters, since it allows the computation of the Earth's perturbative influence on an asteroid orbiting the Sun.

The KM equations are described in orbital elements using the Lagrange planetary equations. The disturbing function $\mathcal{R}$ in Equation 2 is based on the expression of the Hamiltonian of the three-body problem in a synodic reference frame, using to a series expansion with the mass of the third body as the small parameter:

$$
\mathcal{R}=-\mu\left(\frac{1}{r}+\frac{\cos \theta}{r^{2}}-\frac{1}{\sqrt{1+r^{2}-2 r \cos \theta}}\right)
$$

where $\mu$ is the normalized gravitational parameter, $\mathrm{r}$ is the distance to the barycenter and $\theta$ is the angle formed by the vectors connecting the third body to the primary and the secondary [34].

This method can then be used to compute the changes in the orbital elements of the asteroid, caused by the Earth's gravitational influence. This calculation is made at each of its periapsis passages $(v=0)$; these changes are then added to the previously known orbital elements to obtain the updated motion. In this way, the action of the KM can be represented by the mapping $\mathcal{K}$ :

$$
\mathcal{K}:\left\{a, e, i, \omega \mid \alpha_{P_{n}}\right\} \mapsto\left\{\Delta a, \Delta e, \Delta i, \Delta \omega \mid \alpha_{P_{n+1}}\right\}
$$

where $a, e, i, \omega$ are the commonly denominated orbital elements and the parameter $\alpha_{P}$ represents the phasing of the perturbed body with the one provoking the disturbance at the motion's periapsis. In contrast, the variable $\alpha$ is used to account for the phasing at an arbitrary point in the orbit: it is the angle in between the Sun-Earth line and the projection of the Sun-asteroid line in the ecliptic plane, using the synodic reference frame. This variable is computed as a function of the longitude of the ascending node $\Omega$ [34], but is used here instead for a better visualization of the problem.

The equations that yield the mapping $\mathcal{K}$ are given by Picard's first iteration on the previously mentioned Lagrange planetary equations: 


$$
\begin{aligned}
\Delta a & =\frac{2}{n a} \int_{-\pi}^{\pi} \frac{\partial \mathcal{R}}{\partial v} d v \\
\Delta e & =\frac{1-e^{2}}{n a^{2} e} \int_{-\pi}^{\pi} \frac{\partial \mathcal{R}}{\partial v} d v-\frac{\sqrt{1-e^{2}}}{n a^{2} e} \int_{-\pi}^{\pi} \frac{\partial \mathcal{R}}{\partial \omega} d v \\
\Delta i & =-\int_{-\pi}^{\pi} \frac{1}{n a^{2} \sqrt{1-e^{2}} \sin i} \frac{\partial \mathcal{R}}{\partial \Omega}-\frac{\cos i}{n a^{2} \sqrt{1-e^{2}} \sin i} \frac{\partial \mathcal{R}}{\partial \omega} \\
\Delta \omega & =\int_{-\pi}^{\pi} \frac{\sqrt{1-e^{2}}}{n a^{2} e} \frac{\partial \mathcal{R}}{\partial e}-\frac{\cos i}{n a^{2} \sqrt{1-e^{2}} \sin i} \frac{\partial \mathcal{R}}{\partial i} \\
\Delta \alpha & =2 \pi\left(\sqrt{\frac{a_{n+1}^{3}}{1-\mu}}-1\right)
\end{aligned}
$$

The $\mathrm{KM}$ is not computed throughout the entire motion; instead, it is used inside a neighboring region of the Earth, within which its gravitational perturbation is non-negligible. This region is defined by an interval of $\alpha$ values, shown to capture the extent of the disturbing effect; outside it, the gravitational perturbation of the Earth is so small that can be effectively neglected. In addition, as a perturbation model, the KM requires the asteroid to be outside the Earth's sphere of influence to be accurate; otherwise, some of the approximations that its equations entail cease to be valid. In this case, other models can be considered, such as Öpik's method [26, 35, 36] or the pseudostate technique [37, 38]. From a planetary protection point of view, this condition also acts as a safety measure, as it binds the asteroid's motion to a region in which the collision risk with the Earth is mitigated.

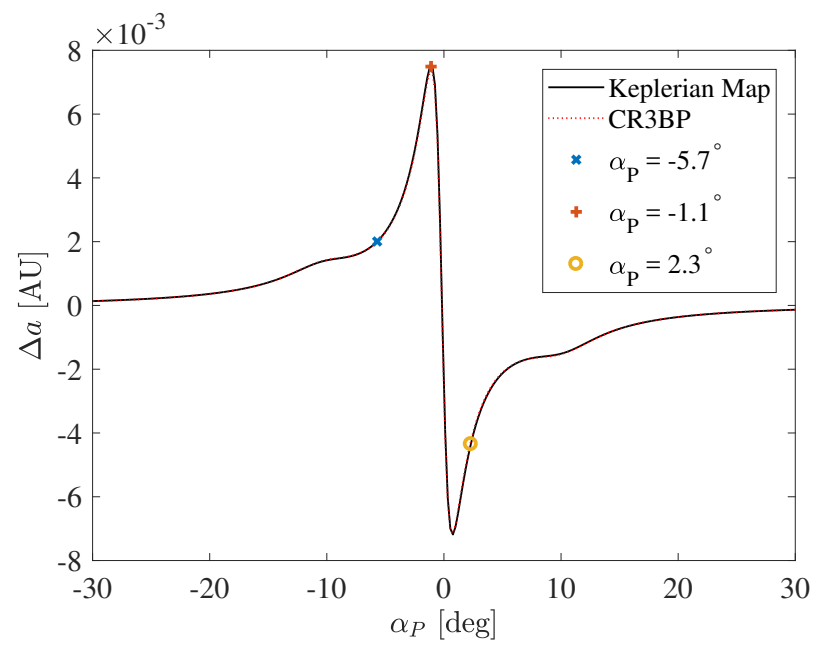

Fig. 1 Kick-map: change in $\Delta a$ with $\alpha_{P}$ for asteroid 2016 RD34

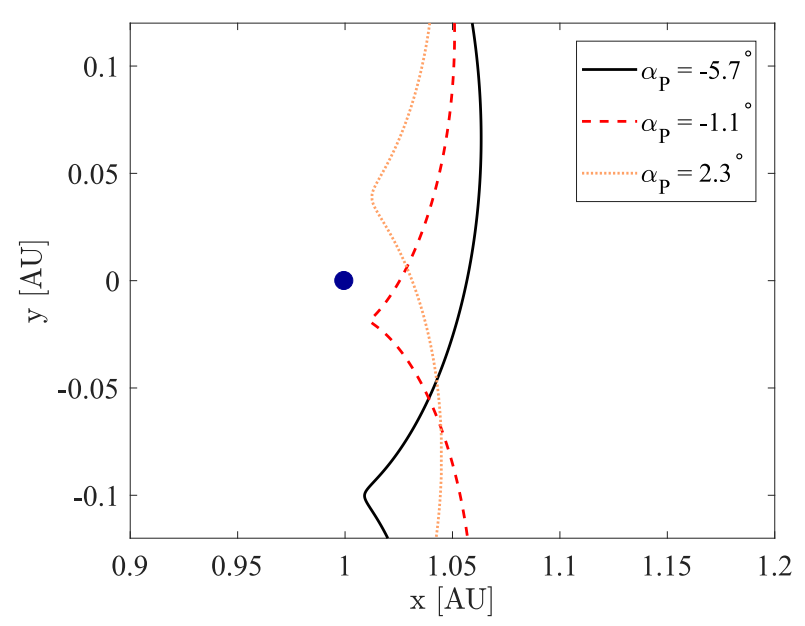

Fig. 2 Three different Earth encounters of asteroid 2016 RD34

An interesting application of the KM is the kick-map, a visual representation of the orbital elements' variation as a function of $\alpha_{P}$. As an example, Figure 1 shows the kick-map representing the semi-major axis change as a function of the phasing $\alpha_{P}$, for asteroid 2016 RD34. Three points are highlighted, corresponding to the particular changes undergone by three different possible encounters shown in Figure 2(Earth's radius is scaled to the size of Hill radius, for visibility). It is worth noting that the semi-major axis function is also computed using the CR3BP, as shown on Figure 1 
The corresponding plot overlaps with the kick-map computed with the KM, reporting a very good accuracy for the latter model.

\section{Design of Earth-Resonant Captures}

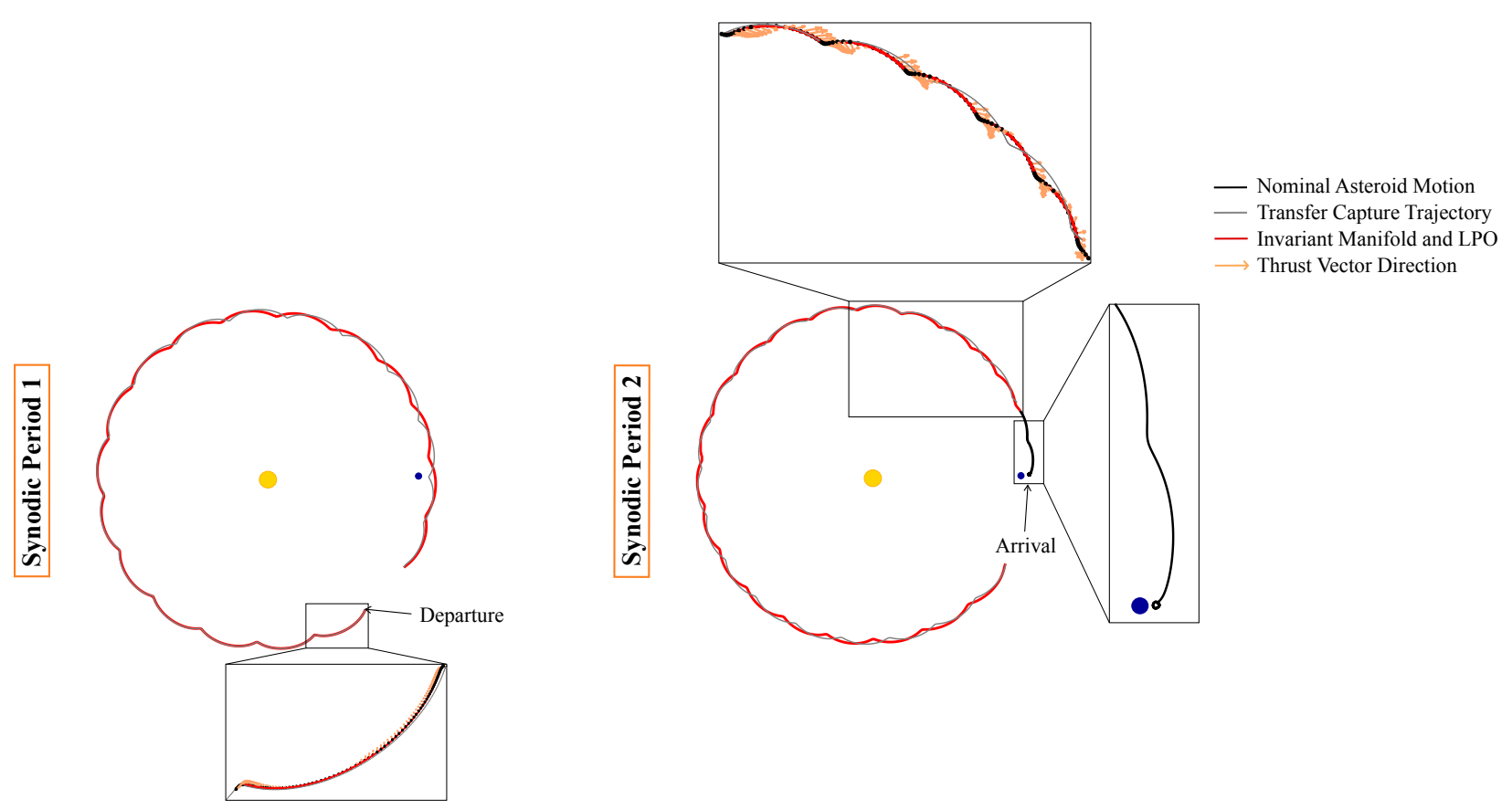

Fig. 3 Earth-resonant capture trajectory for asteroid $2011 \mathrm{MD}$ in the synodic reference frame, divided in two sequential synodic periods for clarity

This section summarizes the steps involved in the design of an Earth-resonant capture trajectory using the multi-fidelity dynamical framework.

Figure 3 illustrates the end product of the multi-fidelity design: a low-thrust nearly resonant Earth capture. The term resonant is here loosely used to account for two consecutive encounters with the Earth. These encounters, which correspond to the asteroid's closest approaches to the Earth, occur outside the planet's sphere of influence. Nevertheless, their geometry is ultimately defined by a strict relation between the orbital periods of the Earth and the asteroid. The trajectory arrives to a stable invariant manifold orbit connected to an LPO after two sequential synodic periods, wherein each includes one Earth encounter. The two zoomed out arcs of Figure 3 depict the thrusting segments; for the remaining orbits, the asteroid is coasting (Earth's radius is increased for better visualization).

The entire multi-fidelity dynamical framework, trajectory design and asteroid selection processes can be observed in the flowchart on Figure 4 from the initial asteroid filtering to the multi-fidelity high-thrust and low-thrust trajectory designs.

Following Figure 4 the pruning process detailed in Sub-section III.A starts by obtaining NEA's ephemerides from the Minor Planet Center (MPC) website [39]. An estimate of the capture $\Delta v_{I}$ is made using a filter, and all asteroids 


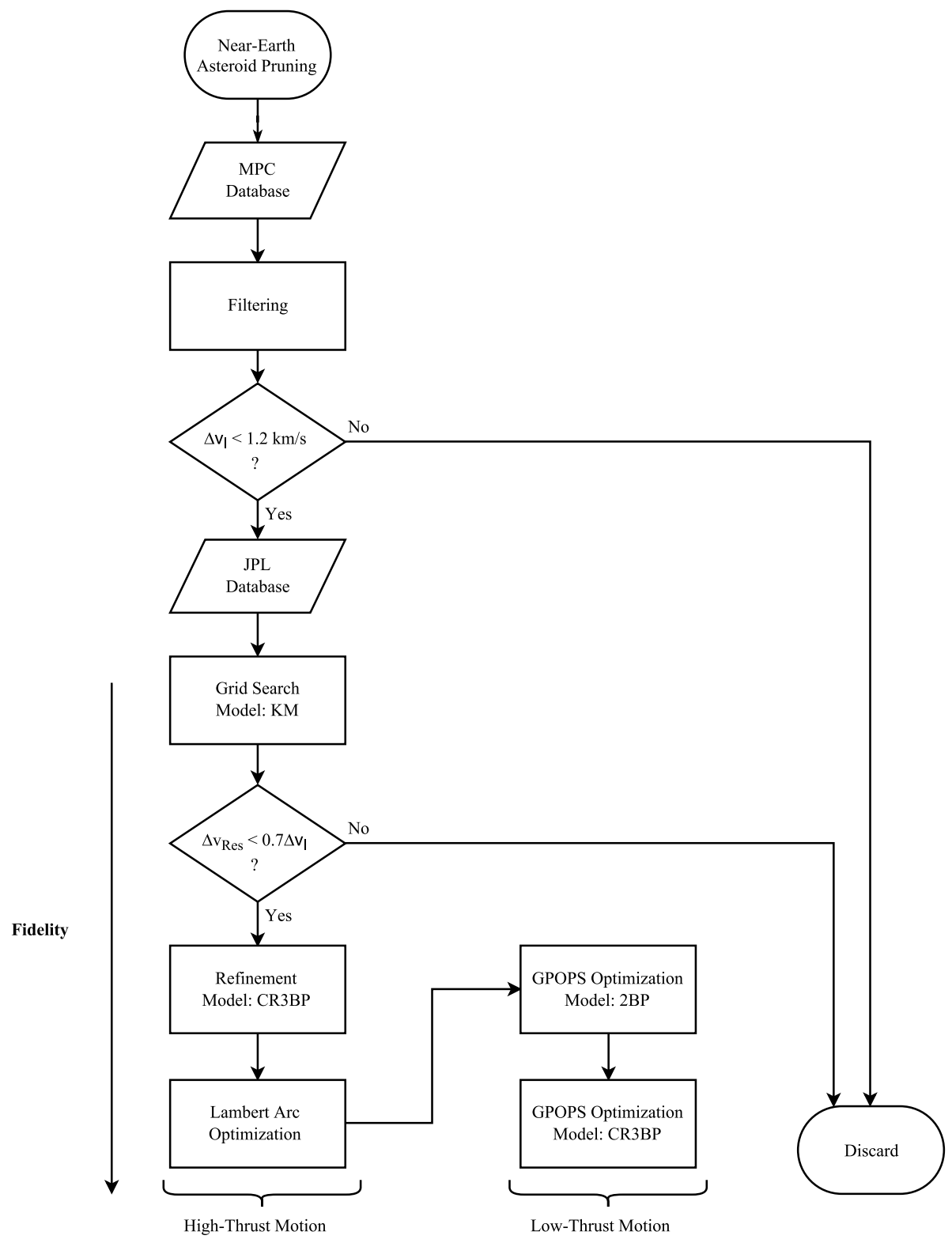

Fig. 4 Flowchart detailing the proposed multi-fidelity design of resonant captures

whose capture cost does not comply with a threshold are discarded.

Subsequently, detailed orbital parameters are obtained from the Horizons JPL database [40] and the multi-fidelity high-thrust design starts, as expanded in Sub-section III.B First, the computation of the resonant capture is made for the current asteroid pool, using the KM as the model of motion. Then, the trajectory for the remaining asteroids is refined in the CR3BP. Lastly, the filter estimate of the capture cost $\Delta v_{I}$ is replaced by a Lambert arc optimization. The obtained trajectory is used as a first guess for the low-thrust optimization, which is further explained in Section IV.

It is important to highlight the difference between the Earth-resonant design and a direct capture trajectory. For the latter, the asteroid is moved from its initial orbit directly into the invariant manifold connected to the LPO, without 
exploiting previous Earth's approaches. As such, a direct capture requires only one maneuver: the manifold insertion, henceforth referred to as $\Delta v_{I}$.

\section{A. Asteroid Pruning}

In order to select the asteroids in which the proposed Earth-resonant trajectory would show the greatest advantages, the entire population of registered NEA was considered for the initial asteroid pool. Their orbital elements, as of June, 2018, were obtained from the MPC website.

From this pool, the targets deemed unfit were pruned out. In order to do so, a filter was employed. It computes an estimate of $\Delta v_{I}$ based on an analytical bi-impulsive transfer approximation to each invariant manifold trajectory, returning the minimum cost and corresponding orbit. The filter has been shown to act as a lower threshold to a Lambert arc capture cost, while still being a good approximation for it [22]. Thus, its main purpose is to work as a fast decision-maker to rule out clearly unfit targets. As indicated in Figure 4, it is only used in a preliminary stage, being later replaced by a Lambert arc optimization.

For completeness, this section includes a brief summary of how the filter works, as first described by Sánchez et al. [20] and later expanded upon [22]. The bi-impulsive maneuver implemented here considers one burn on the perihelion and one on the aphelion, in which only one of the two is responsible for an inclination correction, and both include a semi-major axis change. This is described by Equation 5 .

$$
\Delta v_{I}=\sqrt{\Delta v_{a 1}^{2}+\Delta v_{i 1}^{2}}+\sqrt{\Delta v_{a 2}^{2}+\Delta v_{i 2}^{2}}
$$

in which $\Delta v_{a}$ is the classical change in semi-major axis maneuver, whereas $\Delta v_{i}$ is the inclination change. Thus, there are four computed values for $\Delta v_{I}$, depending on whether the perihelion or aphelion burn is the first and which of them will include the inclination correction; the lowest value out of these will be the filter output.

The filter was then used to estimate the direct capture cost for each candidate; a rough limit of $\Delta v_{I}=1.2 \mathrm{~km} / \mathrm{s}$ was imposed, as it left already a large pool of asteroids for study, while pruning out those whose capture fuel would clearly be too high for a feasible mission.

Posteriorly, the detailed ephemerides from this reduced list were taken from the Horizons JPL database [40], representing the period between 2020 and 2100. There are, in this time-frame, several synodic periods which can be selected for capture, and a careful choice yields the lowest $\Delta v_{I}$. The preferred period is the one in which the asteroid experiences the greatest change in orbital elements when encountering the Earth. This decision comes from the assumption that this change corresponds to a greater sensitivity to the Earth's perturbation and, therefore, to the highest optimization sensitivity. 


\section{B. Multi-Fidelity High-Thrust Design}

After the reduced asteroid list is obtained, the process to develop the multi-fidelity high-thrust trajectory is applied to each candidate. This motion is used as an initial guess for the low-thrust optimization of the trajectory, which can be visualized on Figure 3. The full motion can be divided in four different phases, which are highlighted in Figure 5 and further explained here:

Phase A starts when the asteroid is at the first periapsis right outside the perturbative region of influence of the Earth; at this point, the asteroid's velocity is changed by $\Delta v_{M}$, altering its path. In the low-thrust capture motion, this maneuver corresponds to a short thrusting period in the beginning of the motion. This can be seen in Figure 3 . in the highlighted section in Synodic Period 1.

Phase B corresponds to the first encounter with the Earth, in which the asteroid is affected by its perturbation. This region is defined by $|\alpha|=\frac{\pi}{8}+\frac{\Delta \alpha_{P}}{2}$, which delimits a sufficiently large zone to encompass all $\alpha_{P}$ in which the object's motion is noticeably perturbed. In Figure 5, the region is marked by the diagonal lines forming a conical region in space.

Phase $C$ consists on the motion in between Earth encounters, including the maneuver to insert the asteroid into an invariant manifold connected to an LPO, with a cost given by the variable $\Delta v_{I}$. In the low-thrust trajectory, this maneuver equates to a short thrusting period before insertion into the LPO. This can be seen on Figure 3 , in the highlighted section in Synodic Period 2.

Phase D culminates with the second Earth encounter, where the asteroid moves from the invariant manifold where it was inserted into the LPO.
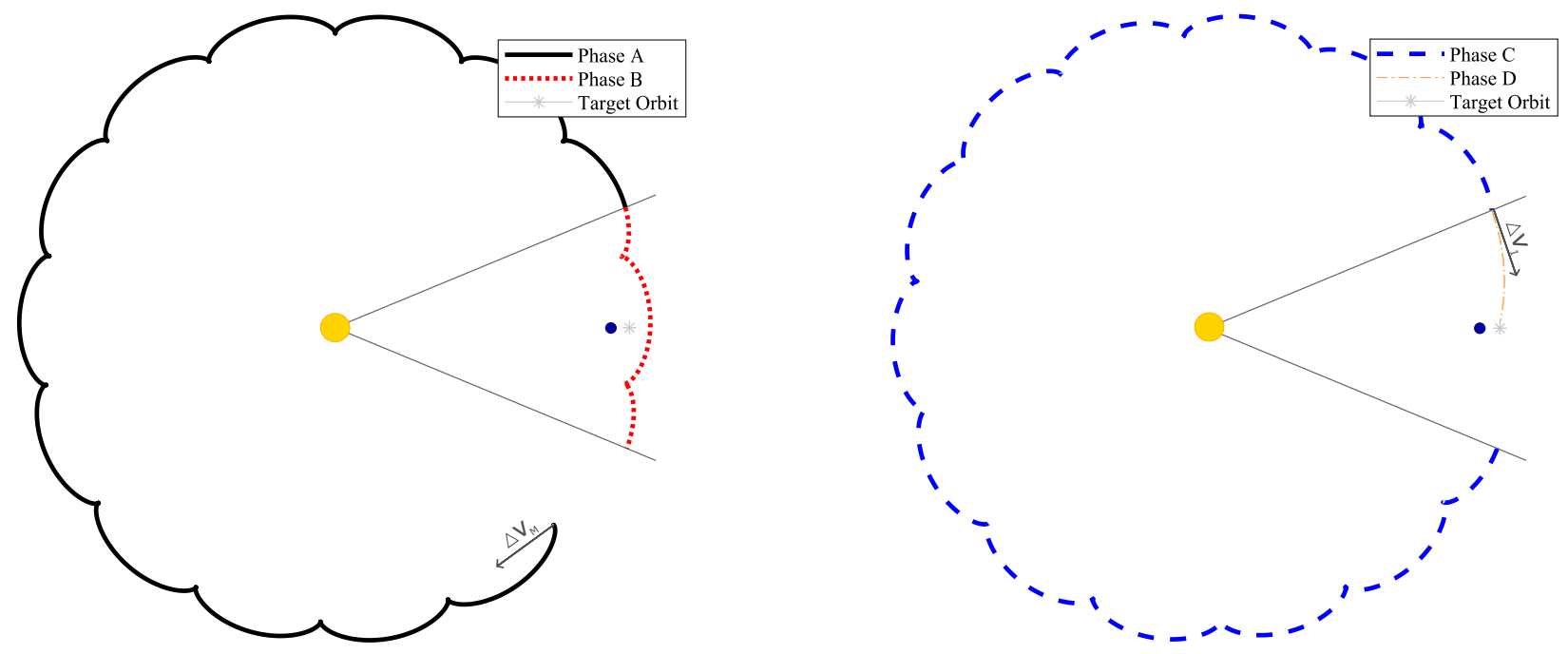

Fig. 5 Phases of the Earth-resonant capture trajectory in sequence. The direction of the motion represents an asteroid of semi-major axis $>1$

The dynamics in Phases A and C are modeled with a simple two-body problem, with the Sun exerting the central 
gravitational attraction; its path is only altered by an initial $\Delta v_{M}$, and a $\Delta v_{I}$ at the end of the latter. However, due to the close proximity to the Earth, its perturbation cannot be neglected in Phase B, and both the KM and CR3BP will be used to model its dynamics. Finally, the invariant manifold dynamics in Phase D are modeled using the CR3BP, as described by previous publications by Sánchez et al. [22].

The final capture $\Delta v_{C}$ is the added total of the two different maneuvers, shown in Equation 6 for the resonant and direct cases - the latter of which, as previously mentioned, only consists of Phases C and D.

$$
\Delta v_{C}= \begin{cases}\Delta v_{M}+\Delta v_{I}, & \text { Resonant Capture } \\ \Delta v_{I}, & \text { Direct Capture }\end{cases}
$$

The multi-fidelity framework for the high-thrust motion is then divided into three steps, distinctive due to their different dynamical models and increasing complexity. The resulting trajectory acts as an initial guess to reach a low-thrust controlled solution.

For the Grid Search and the Refinement steps, the capture trajectory for Phase B is modeled respectively in the KM and in the CR3BP. The $\Delta v_{I}$ cost from Phase $\mathrm{D}$ is estimated by the filter described in Equation 5, which is later replaced by a Lambert arc optimization. As discussed in Sub-section III.A the filter is useful to obtain a quick estimate of the transfer costs, so that the initial optimization can focus on exploiting Earth's perturbation. Nevertheless, future work could consider more accurate approaches to obtain $\Delta v_{I}$, such as a fast Lambert arc estimator [41].

It is important to denote that the goal, when developing this high-thrust trajectory, is to achieve the smallest possible $\Delta v_{C}$; according to the Tsiolkovsky equation, this will correspond to the highest retrievable mass for a fixed amount of propellant.

\section{Step 1: Grid Search}

In this step, the modeling of the Earth encounter in Phase B is made using the KM, with the capture cost $\Delta v_{C}$ computed as the sum of $\Delta v_{M}$ and the filter estimate for $\Delta v_{I}$. The purpose of implementing $\Delta v_{M}$ in the beginning of the transfer is to ensure that the orbital changes occurring during the Earth encounter, due to the Earth's perturbation, are optimal: the KM is used for a quick assessment of how the asteroids' positioning relative to the Earth will impact their orbits.

As such, the grid search is performed by computing $\Delta v_{C}$ for values of $\Delta v_{M}$ from approximately $-50 \mathrm{~m} / \mathrm{s}$ to $50 \mathrm{~m} / \mathrm{s}$, with a step of $0.02 \mathrm{~m} / \mathrm{s}$ : values that cause significant change in the final capture cost, while being comparatively very small. This is done in the following order: each computed motion starts with a change in orbital elements provoked by their respective $\Delta v_{M}$. Then, the KM equations are used to calculate the $\{\Delta a, \Delta e, \Delta i, \Delta \omega\}$ set of orbital element changes for each case. Finally, a grid is obtained, with the $\Delta v_{C}$ cost as computed on Equation 6 as a function of $\Delta v_{M}$. This grid, 
together with the minimum value for $\Delta v_{C}$, can be seen in Figure 6 for asteroid 2016RD34.

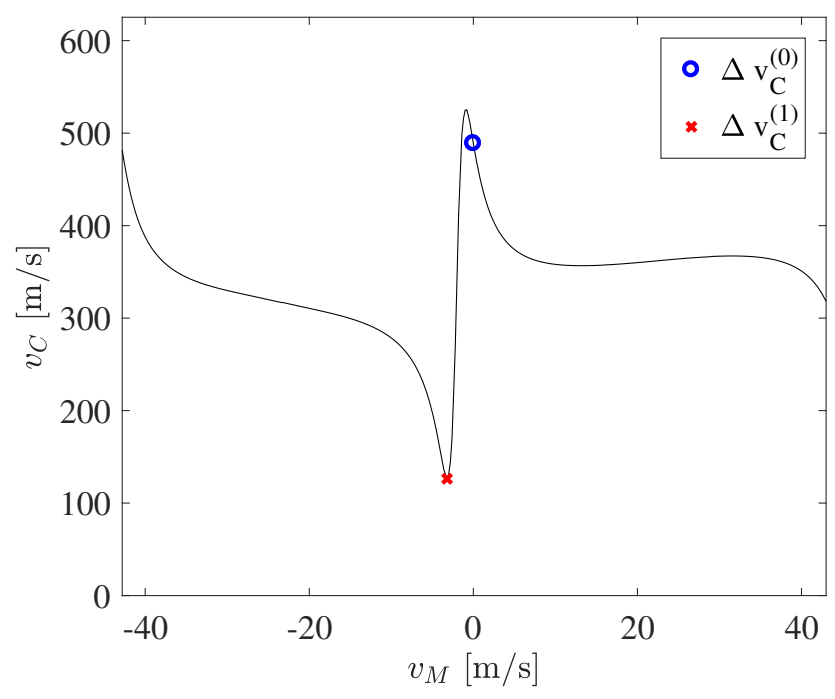

Fig. 6 Grid search for asteroid 2016RD34

Using this grid, the smallest capture cost value and its corresponding initial maneuver are retrieved. On Figure 6 . this value is highlighted with a red cross. Throughout this paper, these have the respective denominations of $\Delta v_{C}^{(1)}$ and $\Delta v_{M}^{(1)}$. These are the values to pass on to the next section, Refinement.

On Figure 6 it can be seen that, by performing no maneuver (i.e. $\Delta v_{M}=0$, represented by $\Delta v_{C}^{(0)}$ ), the capture $\Delta v_{C}$ would be much higher in the case of asteroid 2016RD34. This is a good example of a case in which a very small $\Delta v_{M}$ decreases immensely the final capture value, demonstrating the value of optimizing the Earth encounter.

One simplification must be mentioned: although $\Delta v_{M}$ will cause a change in the orbital elements before the first Earth encounter, this change is so small that makes a negligible difference when computing the KM perturbation as a function of the asteroid's phasing. In this way, the mapping shown on Equation 3 needs only to be determined for the initial set of orbital elements in an adequate range of $\alpha$. The $\alpha_{P}$ of each motion in the grid can be interpolated out of this computation.

Another point to be made is that, if the absolute value of $\Delta v_{M}$ is increased without any bounds, the asteroid's orbit is eventually going to move forwards or backwards one epicycle, causing $\alpha_{P}$ to be the same. Thus, the analysis is restricted to $\Delta v_{M}$ inside limits that correspond to the asteroid moving backwards or forwards only one epicycle. These limits are further defined after the rough estimate of $50 \mathrm{~m} / \mathrm{s}$ previously mentioned; for the actual computation, the Gauss' form of the variational equations are employed, as following:

$$
\Delta v_{M}=\frac{\mu_{S u n} \Delta a}{2 a^{2} v_{P}}
$$

where $\mu_{\text {Sun }}$ is the Sun's gravitational parameter, $v_{P}$ is the velocity at the periapsis and $\Delta a$ represents the variation in 
initial semi-major axis corresponding to the addition of one epicycle to the asteroid's motion.

\section{Step 2: CR3BP Refinement}

While the KM is able to accurately represent the underlying gravitational disturbance caused by the Earth, resulting in a reduction of capture costs, other non-linear effects may completely distort the sought outcome when passing the solution to the CR3BP. In this way, the solution yielded by Step 1, using the previously optimal $\Delta v_{M}$, needs to be refined to achieve a similar behavior and capture cost.

In general terms, the closer the asteroid is to the perturbing body, the greater its influence on it becomes; as such, the object may undergo several periapsis passages in the region of the Earth's perturbation, but it can be roughly assumed that the one that will exert the most significant impact on its motion will be the one in which $\alpha_{P}$ is the closest to zero. Following this logic, in order to get a similar orbital change using the CR3BP as the one obtained in the previous step with the KM, the closest of its periapsis passages, $\alpha_{\text {closest }}$, should be the same in both models.

In order to target the same $\alpha_{\text {closest }}$, a differential corrector was utilized, which is now explained. This tool will compute the value of $\Delta v_{M}$ that yields the encounter with the intended $\alpha_{\text {closest }}$. This value will be henceforth referred to as $\Delta v_{M}^{(2)}$, while the resulting capture cost will be, analogously, $\Delta v_{C}^{(2)}$.

Differential Corrector A differential corrector is a numerical algorithm purposed to solve the equations of motion of the problem in such a way as to reach a final intended state, in the following manner:

$$
\eta_{f}=f(\eta, t)
$$

where $\eta$ is the body's state vector, with $\eta_{f}$ being the final desired state.

For the case in which $\eta$ describes a six dimensional state vector of position and velocity $(\eta=\{x, y, z, \dot{x}, \dot{y}, \dot{z}\})$, many differential correctors have been described in literature (e.g. [42]). The mathematical development of such a tool is well known: defining the relationships between the initial state vector and a nearby-one as:

$$
\eta(t)=\eta_{0}(t)+\delta \eta(t)
$$

and expanding about the reference solution in a Taylor series, the following equation appears:

$$
\delta \dot{\eta}=A(t) \delta \eta
$$

where $A$ is the Jacobian, or sensitivity matrix of $f$. Then, the solution to Equation 9 is:

$$
\delta \eta(t)=\Phi\left(t, t_{0}\right) \delta \eta\left(t_{0}\right)
$$


which describes the changes in the final state vector as dependent on the initial one, as well as its state transition matrix $\Phi$.

A differential corrector can also be developed for the case in which the state vector is expressed as orbital elements $(\eta=\{a, e, i, \Omega, \omega, M\})$. This proposal employs the semi-analytical approach developed by Alessi and Sánchez [34].

Instead of considering the differential correction of all six orbital elements, the computation is based on the premise to change only one: the mean anomaly $M$, which, assuming the dependencies to other variables to be negligible, will provide a much faster computation. The mean anomaly change as a function of the initial semi-major axis can be described by Equation 12 .

$$
\delta M_{f}=\Phi \delta a
$$

where $\dot{\Phi}=A \Phi$. For these parameters, the Jacobian $A$ can be simply described as

$$
A(t)=\frac{\delta \dot{M}}{\delta a}
$$

The equation that yields $\dot{M}$ is further expanded here:

$$
\begin{aligned}
\dot{M}= & \frac{d}{d t} n\left(t_{f}-t_{0}\right)+\frac{2 \mu}{n a}\left[\frac{1-e^{2}}{1+e \cos v}\left(-\frac{1}{r^{2}}-\frac{2 \cos \theta}{r^{3}}+\frac{r-\cos \theta}{\left(1+r^{2}-2 r \cos \theta\right)^{3 / 2}}\right)\right. \\
& \left.+\frac{3 M \Theta}{2 n}\left(\frac{1}{r^{2}}-\frac{r}{\left(1+r^{2}-2 r \cos \theta\right)^{3 / 2}}\right)\right] \\
& +\frac{\mu\left(1-e^{2}\right)}{n e a^{2}}\left[\left(\frac{1}{r^{2}}+\frac{2 \cos \theta}{r^{3}}-\frac{r-\cos \theta}{\left(1+r^{2}-2 r \cos \theta\right)^{3 / 2}}\right)\left(\frac{2 e a+r \cos v}{1+e \cos v}\right)\right. \\
& \left.+\frac{\Theta \beta}{n}\left(\frac{1}{r^{2}}-\frac{r}{\left(1+r^{2}-2 r \cos \theta\right)^{3 / 2}}\right)\right]
\end{aligned}
$$

where $\Theta=\sin \Omega_{\text {rot }} \cos (\omega+v)+\cos \Omega_{r o t} \sin (\omega+v) \cos i, \beta=-\sin E\left(1+\frac{1-e \cos E}{1-e^{2}}\right), r=\frac{a\left(1-e^{2}\right)}{1+e \cos v}$ and $n$ is the mean motion.

\section{Step 3: Lambert Arc Optimization}

Finally, the $\Delta v_{I}$ guess established by the filter for Phase D is replaced by the computation of a Lambert arc, adding another fidelity level to the framework.

The design of an orbital transfer requires the determination of the specific orbit that goes through two points in space, at a certain time. The task of finding the orbit is called Lambert's Problem, which is the boundary solution for the differential gravitation equation of the two-body problem below: 


$$
\ddot{\overrightarrow{\mathbf{r}}}=-\mu_{C} \cdot \frac{\overrightarrow{\mathbf{r}}}{\|\mathbf{r}\|^{3}}
$$

in which $\vec{r}$ is the position vector and $\mu_{C}$ is the central body's gravitational parameter.

A solution to the Lambert's problem is called a Lambert arc, a trajectory that connects two ephemerides. As an example, in order to devise a trajectory from Earth to Mars, one can fix a calendar date and take the positions of the planets to compute the appropriate Lambert arc; however, an optimizer can be used to determine the date at which the transfer is the most cost-effective. In this paper, this optimization problem is tackled by EPIC [43], a global trajectory optimizer. EPIC performs domain decomposition, where each domain is evaluated based on the evolution of a population of agents; its purpose is to generate a series of very good local optima instead of a global one, such that there is more flexibility to the mission design.

This Lambert arc connects one of the invariant manifold trajectories to the asteroid's trajectory after the first encounter with the Earth. EPIC is then employed to compute several arcs, depending on the number of orbital revolutions chosen-in this case, the maximum value was 4 . The solution with lowest $\Delta v_{I}$ is the one chosen for the design, except in cases where the time of flight of the computed arc was too small for the low-thrust trajectory to be feasible.

\section{Low-Thrust Asteroid Hauling}

A low-thrust propulsive system is characterized by generally having an $I_{\mathrm{SP}}$ at least ten times higher than a chemicalthrust one. Therefore, it uses less propellant for the same amount of spacecraft mass. This makes the use of low-thrust engines very attractive for asteroid retrieval, when the combined spacecraft-asteroid system's mass can be very high.

Thus, the computation of the low-thrust trajectory required for asteroid hauling starts with the multi-fidelity model previously utilized. The considered initial setting is akin to the one used by NASA's Asteroid Redirect Robotic Mission concept [8]: a spacecraft of 5,500 kg dry mass and 10 tonnes of propellant, using a high power solar electric propulsion system of roughly $40 \mathrm{~kW}$ and $I_{\mathrm{SP}}$ of $3,000 \mathrm{~s}$, yielding a maximum thrust capability of $2 \mathrm{~N}$.

For each phase of the optimization, the asteroid's mass that can be hauled by the low-thrust engine is heavily dependent on the thrust vector, which is the decision variable of the optimization. The entire system's mass can only be computed after solving the optimal control problem. An initial guess for this can be estimated, using the chemical-thrust $\Delta v_{C}$ solution and the Tsiolkovsky equation. This mass will prove to be quite far from the actual value; in order to correct it, a mass homotopy will be performed, which will be explained in Sub-section IV.C. Since the spacecraft's fuel and dry masses are fixed, maximizing the system's mass is equivalent to doing so for the carried asteroid mass. 


\section{A. Mass Considerations}

In order to establish the feasibility of the mission, the spacecraft must be able to carry its own weight, the fuel mass and the asteroid's. This is described by:

$$
m_{\mathrm{ast}}+m_{\mathrm{dry}}+m_{\mathrm{fuel}}=m_{\mathrm{opt}}
$$

where $m_{\mathrm{dry}}$ and $m_{\text {fuel }}$ are already established as respectively 5.5 and 10 tonnes, and $m_{\mathrm{opt}}$ is the output result of the optimal control problem, representing the entire system's mass. The asteroid mass that can be hauled by the spacecraft, $m_{\text {ast }}$, can be computed by solving Equation 15 , once $m_{\mathrm{opt}}$ is known.

\section{B. Optimal Control Problem}

The conversion from the high-thrust trajectory into a low-thrust motion is done by formulating an optimal control problem, where the thrust is the control variable and the objective is to maximize the final system's mass (after the propellant is depleted), while constrained by the motion's boundaries and position targets. In order for this to be computationally solvable, the optimal control problem has to be transformed into a non-linear programming problem (NLP). GPOPS-II is the software employed for this purpose [44]; it uses orthogonal collocation to transform the continuous problem into a discrete one, which is posteriorly tackled by an NLP solver-IPOPT, an interior point optimizer [45]. The applied collocation method is a Legendre-Gauss-Radau (LGR) scheme: the solution is given by a polynomial that can fit the continuous problem in selected points, called the collocation points: the dynamics are solved in these, and they are evaluated against the candidate Legendre polynomials.

Since GPOPS-II is an hp-adaptive method, both the number of mesh intervals (p) and the degrees of the polynomials in question (h) can be chosen for optimal performance. The way in which GPOPS-II is set up to manipulate these parameters can be found in [46, 47].

The optimal control problem to be tackled is defined in the general form: to determine the state $x(t)$, control $u(t)$, initial time $t_{0}$, final time $t_{f}$, integrals $q$ and the static parameters $s$ that minimize the cost function:

$$
J=\Phi\left[\mathbf{x}\left(t_{0}\right), t_{0}, \mathbf{x}\left(t_{f}\right), t_{f}, q, s\right]
$$

subject to the dynamic constraints

$$
\dot{\mathbf{x}}=f[\mathbf{x}(t), \mathbf{u}(t), t, s]
$$

the event constraints

$$
b_{\min } \leq b\left[\mathbf{x}\left(t_{0}\right), t_{0}, \mathbf{x}\left(t_{f}\right), t_{f}, q, s\right] \leq b_{\max }
$$


the inequality path constraints

$$
c_{\text {min }} \leq c[\mathbf{x}(t), \mathbf{u}(t), t, s] \leq c_{\max }
$$

and the integral constraints

$$
q_{\text {min }} \leq q \leq q_{\text {max }}, q_{i}=\int_{t_{0}}^{t_{f}} Q_{i}[\mathbf{x}(t), u(t), t, s] d t
$$

This general problem is then adapted to fit the low-thrust trajectory case. As already stated, the decision variable will be the thrust vector throughout the motion; starting from the initial time and state, this variable is optimized in such a way that the final system's mass is the highest. Each state is computed using the equations of motion of the problem coupled with the acceleration provided by the thrust, forming the dynamical constraints.

A multi-fidelity model, going from a two-body equinoctial system to the CR3BP, was implemented to find a solution to the optimal control problem. The selection of the two-body equinoctial system allows a straightforward definition of the states' bounds, guaranteeing an improved convergence. Furthermore, this description avoids the singularities that could be encountered when dealing with easily retrievable NEAs [21], whose orbits are frequently quasi-circular and quasi-planar. The parameters for the two-body system are presented in Equation 21] [48].

$$
\begin{aligned}
\dot{p} & =\frac{2 p}{w} \sqrt{\frac{p}{\mu}} \frac{T_{\theta}}{m} \\
\dot{f} & =\sqrt{\frac{p}{\mu}}\left[\sin (L) \frac{T_{\theta}}{m}+\frac{1}{w}[(w+1) \cos (L)+f] \frac{T_{\theta}}{m}-\frac{g}{w}(h \sin (L)-k \cos (L)) \frac{T_{n}}{m}\right] \\
\dot{g} & =\sqrt{\frac{p}{\mu}}\left[-\cos (L) \frac{T_{r}}{m}+\frac{1}{w}[(w+1) \sin (L)+g] \frac{T_{\theta}}{m}-\frac{f}{w}(h \sin (L)-k \cos (L)) \frac{T_{n}}{m}\right] \\
\dot{h} & =\frac{p}{\mu} \frac{s^{2}}{2 w} \cos (L) \frac{T_{n}}{m} \\
\dot{k} & =\frac{p}{\mu} \frac{s^{2}}{2 w} \sin (L) \frac{T_{n}}{m} \\
\dot{L} & =\sqrt{\mu p}\left(\frac{w}{p}\right)^{2} \frac{1}{w} \sqrt{\frac{p}{\mu}}(h \sin (L)-k \cos (L)) \frac{T_{n}}{m}
\end{aligned}
$$

where $w=\frac{p}{r}=1+f \cos (L)+g \sin (L)$ and $s=1+h^{2}+k^{2}$.

Once a solution is computed using the aforementioned dynamical model, it is used as a first guess for a final optimization in the CR3BP. Using a simplified model as a first solution makes it simpler and faster for the program to converge, as these types of problems are extremely sensitive to the set up and the process is very time consuming.

Each of the models has a few more differences, detailed on Table 1 . The bounds and event constraints are defined using the Lambert arc initial guess and the target state vector.

The computation of the objective function is common for both cases, as shown in Equation 22 
Table 1 Definition of parameters and functions for the two-body and CR3BP optimal control problems

\begin{tabular}{|c|c|c|}
\hline Problem Parameters & $2 \mathrm{BP}$ & CR3BP \\
\hline State Vector $\mathbf{x}$ & $\{p, f, g, h, k, L, w\}$ & $\{x, y, z, \dot{x}, \dot{y}, \dot{z}\}$ \\
\hline Control Vector $\mathbf{u}$ & $\left\{u_{r}, u_{\theta}, u_{n}\right\}$ & $\left\{u_{x}, u_{y}, u_{z}\right\}$ \\
\hline Dynamical Constraints & Equation 21 & Equation 1 \\
\hline Reference Frame & Earth-Centered Inertial & Synodic \\
\hline Objective Function $J$ & & \\
\hline
\end{tabular}

${ }^{*}$ Including the added thrust component

$$
\dot{m}=-\frac{\|\mathbf{T}\|}{I_{\mathrm{SP}} g_{0}}
$$

\section{Mass Homotopy}

The final mass is the optimized parameter of this process. In simple terms, the goal of the optimal control problem is to utilize the propellant in the most efficient way, starting from a given initial system mass (as in Equation 15). This does not, however, change the initial mass. For the first iteration, the mass is computed such that the total thrust time is equal to $1 \%$ of the time of flight. Yet, this value is very far from the capabilities of the low-thrust system engine. As such, the initial mass can be increased after each iteration on GPOPS-II using a continuation method, here described as a mass homotopy.

Thus, at each iteration, the initial mass value is raised in small steps. This increase has to be small enough at each iteration for the optimal control problem to converge quickly using the previous iteration solution as initial guess. As such, the initial mass at each iteration is estimated by means of the Tsiolkovsky equation, using the still available thrust time and propellant mass as tuning parameters.

Finally, the entire low-thrust trajectory is developed as follows: starting from the high-thrust guess, the optimization is performed until a solution is reached. If the difference in between final and initial mass is smaller than 10 tonnes (the propellant), and the total thrust time is shorter than the total transfer time, then the mass homotopy is performed and the optimization occurs again for the new solution; otherwise, the process is terminated.

\section{Results and Discussion}

This section presents the obtained values at each step of the multi-fidelity design process (see Figure 4). The benefits of employing an Earth-resonant transfer for asteroid retrieval are analyzed, and the different fidelity models that make up the framework are compared in terms of accuracy and computational cost. 
Table 2 Dates, times of flight (TOF) and final capture orbit for each asteroid in the candidate list

\begin{tabular}{ccccc} 
Asteroid & Initial Date & TOF $_{\text {Direct }}[\mathrm{y}]$ & TOF $_{\text {Resonant }}[\mathrm{y}]$ & Capture LPO \\
\hline 2016 RD34 & $05-22-2033$ & 12.92 & 35.40 & VL2 $^{a}$ \\
2012 TF79 & $01-02-2020$ & 19.76 & 37.38 & VL2 \\
2011 MD & $01-08-2070$ & 14.73 & 36.73 & VL2 \\
2011 BL45 & $23-12-2072$ & 17.62 & 42.76 & VL2 \\
2014 BA3 & $09-09-2035$ & 10.48 & 25.22 & VL1 $^{b}$ \\
2000 SG344 & $22-01-2033$ & 41.48 & 81.04 & PL2 $^{c}$ \\
2017 FJ3 & $10-03-2047$ & 4.46 & 11.21 & PL2 \\
2017 BN93 & $17-04-2034$ & 13.86 & 47.30 & VL2 \\
2010 VQ98 & $15-01-2063$ & 16.66 & 48.77 & VL2 \\
2008 UA202 & $01-01-2020$ & 29.39 & 40.53 & PL2 \\
2006 JY26 & $01-01-2020$ & 44.05 & 30.93 & HL2 \\
2006 BZ147 & $21-01-2039$ & 38.26 & 58.11 & HL2 \\
\hline
\end{tabular}

${ }^{a}$ Vertical Lyapunov in $\mathrm{L}_{2}$

${ }^{b}$ Vertical Lyapunov in $\mathrm{L}_{1}$

${ }^{c}$ Planar Lyapunov in $\mathrm{L}_{2}$

${ }^{d}$ Halo in $\mathrm{L}_{2}$

\section{A. Asteroid Candidate List}

From the MPC database, over 18,000 asteroids were considered. The filter described in Sub-section III.A was used to prune the ones whose $\Delta v$ with direct capture was higher than $1.2 \mathrm{~km} / \mathrm{s}$. Then, the ephemerides of the remaining 88 asteroids were retrieved from the JPL database. For each candidate, the synodic period with the greatest orbital changes was selected, as described in Sub-section III.A.

To retrieve the minimum $\Delta v$ for a resonant capture, the grid search is performed on each of these NEA. The values are posteriorly compared with the direct capture $\Delta v$ for each asteroid. The selection of candidates for refining and further analysis is based on whether or not the resonant capture $\Delta v$ is smaller than the direct one by at least $30 \%$; this resulted in a total of 12 asteroids. Captures with lesser improvements than this threshold were simply deemed not interesting enough to pursue, given how much longer the transfer time of a resonant capture would become, as compared with the direct option.

Table 2 shows the candidate list asteroids' designations, the times of flight and the date when each transfer begins (chosen as explained in Sub-section III.A.).

\section{B. Trajectory Design}

For each of these asteroids, the values found for every step detailed in Subsection III.B are depicted on Table 3, as well as the values of $\alpha_{\text {closest }}$ to be targeted by the differential corrector. It is important to note that the direct $\Delta v_{C}$ is the optimized Lambert arc cost, as detailed in Sub-section III.B.3. Again, the resonant $\Delta v$ have different denominations 
Table 3 Analysis of $\alpha_{\text {closest }}$ and $\Delta v$ values on each step for the resulting asteroids. The excluded asteroids are pinpointed by reasons ${ }^{a}$ or ${ }^{b}$

\begin{tabular}{cccccc}
\hline Asteroid & $\alpha_{\text {closest }}\left[{ }^{\circ}\right]$ & Direct $\Delta v_{C}$ & \multicolumn{3}{c}{$\operatorname{Resonant} \Delta v_{C}[\mathrm{~m} / \mathrm{s}]$} \\
& & & $\Delta v_{C}^{(1)}$ & $\Delta v_{C}^{(2)}$ & $\Delta v_{C}^{(3)}$ \\
\hline $2016 \mathrm{RD} 34$ & 0.84 & 323.36 & 136.28 & 62.14 & 84.90 \\
$2012 \mathrm{TF79}$ & 0.86 & 272.81 & 94.27 & 83.46 & 72.85 \\
$2011 \mathrm{MD}$ & 0.57 & 206.22 & 64.94 & 54.34 & 138.94 \\
$2011 \mathrm{BL} 45^{b}$ & 2.24 & 377.28 & 47.94 & 60.30 & 339.00 \\
$2014 \mathrm{BA3}{ }^{a}$ & -16.11 & - & - & - & - \\
$2000 \mathrm{SG} 344^{a}$ & 2.60 & - & - & - & - \\
$2017 \mathrm{FJ} 3$ & 1.52 & 1002.3 & 718.26 & 475.08 & 659.31 \\
$2017 \mathrm{BN93}$ & 1.18 & 539.70 & 145.06 & 125.76 & 274.90 \\
$2010 \mathrm{VQ} 98$ & 2.03 & 265.01 & 118.77 & 148.89 & 130.89 \\
$2008 \mathrm{UA202}$ & -4.37 & - & - & - & - \\
$2006 \mathrm{JY} 26^{a}$ & -3.63 & - & - & - & - \\
$2006 \mathrm{BZ}^{a} 47^{b}$ & -26.72 & 1412.10 & 874.29 & 991.70 & 1239.50 \\
\hline
\end{tabular}

${ }^{a}$ Too close to the Earth

${ }^{b}$ Filter underestimation

depending on the step in the multi-fidelity design: $\Delta v_{C}^{(1)}$ corresponds to the KM grid search, $\Delta v_{C}^{(2)}$ to the CR3BP refinement and $\Delta v_{C}^{(3)}$ includes the Lambert arc optimization.

Six asteroids were excluded from the candidate list, for two different reasons. First, the cases of asteroids 2008 UA202, 2006 JY26, 2000 SG344 and 2014 BA3 are particular ones in which the dynamics are too sensitive for an adequate capture trajectory to be computed by the suggested method, as the motion easily enters the Hill radius. These do not have the $\Delta v$ values for each step on Table 3, as the framework did not tackle motions so close to the Earth. The appropriate way to tackle these cases would be to use a model that adequately approximates motions close to the Earth, such as Öpik's method [26, 35, 36] or the pseudostate technique [37, 38].

Second, asteroids 2011 BL45 and 2006 BZ147 are outliers in the sense that, although the capture $\Delta v_{I}$ determined with the filter was shown to fit the criteria, the value resulting from the Lambert arc computed with EPIC was too great to achieve a high capture mass. It was understood that both were cases in which the filter severely underestimated the capture $\Delta v$, and therefore there was no point in pursuing their study. This can possibly be avoided in the future by using a different estimate of $\Delta v_{I}$, such as a Lambert arc estimator or a similar fast transfer maneuver [41].

Even though the filter proves to be an under-estimator for most cases, the efficiency of the resonant trajectory compared to the direct one is kept for most asteroids. After this process, six asteroids remained for the computation of the low-thrust transfer. 
Table 4 Retrievable masses of the candidate asteroids for direct and Earth-resonant captures

\begin{tabular}{ccccc} 
Asteroid & $\mathrm{m}_{\text {Published }}[\mathrm{t}]$ & $\mathrm{m}_{\text {Direct }}[\mathrm{t}]$ & $\mathrm{m}_{\text {Resonant }}[\mathrm{t}]$ & Balance $[\mathrm{t}]$ \\
\hline $2016 \mathrm{RD} 34$ & - & 357 & 1227 & +870 \\
$2012 \mathrm{TF79}$ & $739^{a}$ & 705 & 3161 & +2456 \\
$2011 \mathrm{MD}$ & $800^{b}$ & 784 & 1496 & +712 \\
2017 FJ3 & - & 193 & 293 & +100 \\
2017 BN93 & - & 322 & 521 & +199 \\
2010 VQ98 & $727^{c}$ & 493 & 1515 & +1022 \\
\hline
\end{tabular}

${ }^{a}$ TOF of 7.30 years [22]

$b$ TOF of roughly 6 years [11]

${ }^{c}$ TOF of 8.86 years [22]

\section{Mass Comparison}

As described on Section [V] the chemical-thrust guess was used for the optimization of the low-thrust solution. By iterating through several steps on the mass homotopy process, GPOPS-II achieved the final retrievable masses for the direct and resonant captures. Table 4 shows the comparison between these two values, and also includes some previously published results that assumed similar low-thrust systems.

As expected, the increase in retrievable mass between the direct and resonant trajectories was over $30 \%$ for all cases. The obtained mass values are also much higher than the ones found in literature and, in some cases, allow for the retrieval of over 1000 tonnes of material back to Earth. When comparing the computed low-thrust motion for both models used (2BP with equinoctial elements and CR3BP), the difference in values presented is less than $0.01 \%$, so there was no need to present a distinction.

Figure 7 shows the complete thrust profile of the Earth-resonant capture trajectory for asteroid 2011MD in the CR3BP. It depicts the thrust history throughout time, starting with a small control output corresponding to the $\Delta v_{M}$ maneuver. Then, the asteroid continues coasting until the trajectory reaches the Lambert arc segment, which this proves to be the hardest to optimize as it is associated with the $\Delta v_{I}$ maneuver.

Despite the higher complexity of the CR3BP low-thrust optimal control problem, the final solution features a substantially neat on-off control, indicative that optimality conditions are well satisfied.

\section{Model Comparison}

The KM model was suggested to have a similar accuracy to the CR3BP, coupled with a shorter computational time. In order to support these claims, the results of both models are compared for 88 different sets of initial asteroid orbital elements, and the time spent on each is analyzed. Further comprehensive testings on the KM model can be found in [34].

On Figure 8, the logarithmic mean value of the relative error for the semi-major axis updated with the KM is shown for 300 different $\alpha$ values. The black full-lined plots display the mean error over the 88 asteroids regardless of their 


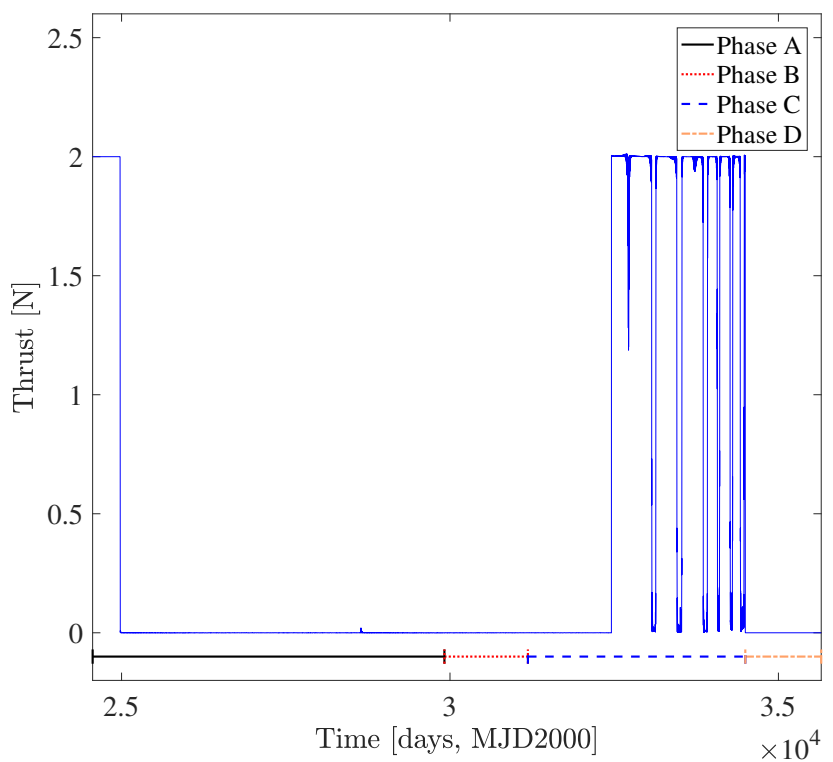

Fig. 7 Thrust profile for the full low-thrust trajectory in the CR3BP as a function of time for asteroid 2011 MD

closest approach to the Earth, while the red dotted plots consider only the ones that never cross the sphere of influence of the planet, totaling 36 bodies. As discussed in Sub-section II.B the relative error is much smaller when the cases that cross the Hill radius are removed: the KM model works best when outside Earth's sphere of influence. Also, the error increase for values close to $\alpha=0$ is correlated to the closest proximity to the Earth, where the Earth's perturbation is the most influential.

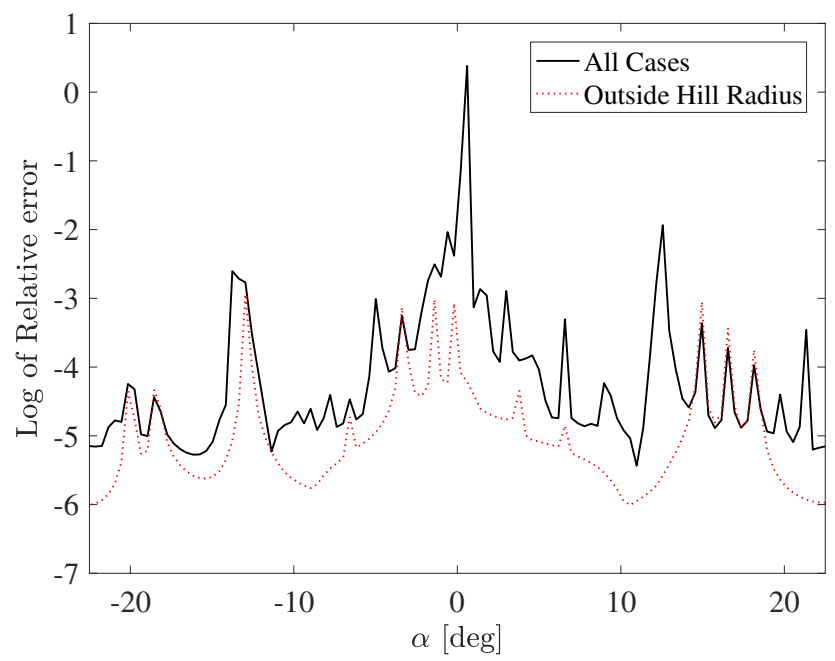

Fig. 8 Logarithm of the mean error for semi-major axis update as a function of $\alpha$

In terms of computational time, both models were used to compute 60 different initial $\Delta v_{M}$ maneuvers for the 88 previously mentioned asteroids. It was found that the KM takes roughly 30 times less computational time than the CR3BP. 
Table 5 Comparison between the maneuver values in the KM (Step 1) and CR3BP refinement (Step 2) models

\begin{tabular}{ccc} 
Asteroid & $\Delta v_{M}^{(1)}[\mathrm{m} / \mathrm{s}]$ & $\Delta v_{M}^{(2)}[\mathrm{m} / \mathrm{s}]$ \\
\hline 2016 RD34 & -3.60 & -2.86 \\
2012 TF79 & 6.42 & 6.58 \\
2011 MD & 1.02 & 1.60 \\
2017 FJ3 & -16.47 & -13.24 \\
2017 BN93 & 6.46 & 7.06 \\
2010 VQ98 & -5.11 & -3.98 \\
\hline
\end{tabular}

Considering the multi-fidelity trajectory design, it was observed that the $\Delta v_{M}$ to reach the same $\alpha_{\text {closest }}$ was very similar in both the KM and the CR3BP, albeit the latter yielding slightly lower $\Delta v_{C}$ results, which can be seen on Table 3 . This allows the inference that the former, being a lower-fidelity framework, models the trajectory nearly as accurately as the higher-fidelity one. The $\Delta v_{M}$ for the KM grid search and the CR3BP refinement can be found on Table 5 they are extremely small, when compared to the overall capture $\Delta v_{C}$.

This comparison allows for the validation of the KM as a relatively accurate model that can provide a good stepping stone for an increase in fidelity in the current framework. The lower computational cost allows for a feasible analysis of the amount of NEA described on this paper, while still presenting very similar results to higher complexity models of motion.

\section{Conclusions}

This paper presents a framework to design nearly resonant trajectories in a multi-fidelity model. The design is carried out in several steps, starting from a high-thrust motion using the KM model and ending in a fully developed low-thrust trajectory in the CR3BP.

This trajectory is applied to the concept of asteroid capture missions, exploiting the orbital perturbations occurring in an Earth-resonant motion to increase the retrievable mass for a list of candidate NEA. These are hauled into LPOs in less than two synodic periods, encountering the Earth twice in their motion.

The retrieved asteroid mass from the resonant trajectory is compared to the one obtained by direct motion and previously published results. Six of them (asteroids 2016 RD34, 2012 TF79, 2011 MD, 2017 FJ3, 2017 BN93 and 2010 VQ98) showed a huge increase with respect to the direct motion and the state of the art. This can be mainly attributed to two decisions: first, the selection of the synodic period with the most advantageous Earth encounter - since each passage will have the asteroid in a different configuration with the planet, distinct results will be obtained; second, the careful exploitation of this encounter with an initial maneuver, to put the asteroid into an optimal insertion orbit. In the end, the cost of this initial maneuver is negligible compared to the insertion cost, but both the former and the Earth encounters are performed mainly to place the asteroid in the best possible orbital configuration at the manifold insertion. Thus, the 
highly increased amount of retrievable mass presented by Earth-resonant trajectories is an extremely good case for their usefulness in asteroid capture missions. These trajectories may become valuable options within a future portfolio of asteroid capture missions.

The main drawback of this method is the increased mission time as opposed to a direct capture. This comes down to a mission design trade-off problem, in which flexibility, time and cost have to be juggled. This method proves to be the most advantageous when the asteroid's mass is too great for any other type of capture to be feasible. Still, mission time can be decreased by scheduling the spacecraft's rendezvous with the asteroid to happen closer to the Earth encounter. Actually, Figure 7 contains a large coasting period (Phase A) that could be easily removed by performing the phasing maneuver much later. However, the cost of this maneuver would grow which, in consequence, would slightly decrease the amount of retrievable mass.

The multi-fidelity framework presented in this paper is very flexible in terms of the desired accuracy, as the user can choose not to undertake all the steps in the process, but instead to stop at wherever it is the most convenient. Plus, the framework can be expanded on fidelity with different tools, depending on the intended application. Future options to include in the framework would be the option to compute trajectories going inside the Hill radius using an alternative to the KM, or a continuation of the design into a full ephemerides model. Nevertheless, the KM proves to be a very good model to approximate the third-body effects while maintaining a low-computational cost, which is very useful for the assessment of multiple trajectory designs. Hence, the framework presented can be adapted to other planetary systems (i.e. Earth-Moon, Jupiter-Europa), and be used for other purposes than asteroid capture, such as moon tours or end-of-life disposal strategies, showing a range of applications worthy of further study.

\section{Acknowledgments}

R.N. would like to acknowledge the support of the Amelia Earhart Fellowship grant and the Coachmakers' Eric Beverley Bursary.

\section{References}

[1] Alvarez, L., Alvarez, W., Asaro, F., and Michel, H., "Extraterrestrial Cause for the Cretaceous-Tertiary Extinction," Science, Vol. 208, No. 4448, 1980, pp. 1095-1108. doi:10.1126/science.208.4448.1095.

[2] Crawford, I., Elvis, M., and Carpenter, J., "The Use of Extraterrestrial Resources to Facilitate Space Science and Exploration,” arXiv preprint arXiv:1605.07691, 2016. doi:10.1093/astrogeo/atw150.

[3] Elvis, M., "Let's mine asteroids-for science and profit," Nature, Vol. 485, No. 7400, 2012, p. 549. doi:10.1038/485549a.

[4] Lewis, J., and Hutson, M., Asteroidal Resource Opportunities Suggested by Meteorite Data, University of Arizona Press, Tucson Arizona, 1993, pp. $523-542$. 
[5] Cole, D., and Cox, D., Islands in Space: The Challenge of the Planetoids, Chilton Books, 1964. doi:10.1126/science.147.3662. 1132.

[6] O’Leary, B., “Asteroidal Resources for Space Manufacturing,” Acta Astronautica, Vol. 6, No. 11, 1979, pp. 1467-1480. doi:10.1016/0094-5765(79)90136-X.

[7] Pelton, J., and Allahdadi, F., Handbook of Cosmic Hazards and Planetary Defense, Springer, 2015. doi:10.1007/978-3-31903952-7.

[8] Brophy, J., Culick, F., Friedman, L., Allen, C., Baughman, D., Bellerose, J., and et al., “Asteroid Retrieval Feasibility Study," Report, Keck Institute for Space Studies, Califonia Institute of Technology, Jet Propulsion Laboratory, 2012.

[9] USA Budget 2017, “A Budget Blueprint to Make America Great Again,” Office of the President of the United States, 2017.

[10] Lo, M., "The Interplanetary Superhighway and the Origins Program,” Aerospace Conference Proceedings, 2002. IEEE, Vol. 7 , IEEE, 2002, pp. 7-7. doi:10.1109/AERO.2002.1035332.

[11] Strange, N., Landau, D., McElrath, T., Lantoine, G., and Lam, T., “Overview of mission design for NASA Asteroid Redirect Robotic Mission concept,” 33rd International Electric Propulsion Conference (IEPC2013), edited by N. A. Jet Propulsion Laboratory and S. Administration, 2013.

[12] Landau, D., Dankanich, J., Strange, N., Bellerose, J., Llanos, P., and Tantardini, M., “Trajectories to NAB a NEA (near-Earth asteroid)," 2013, pp. 3251-3262.

[13] Baoyin, H., Chen, Y., and Li, J., "Capturing Near Earth Objects," Research in Astronomy and Astrophysics, Vol. 10, No. 6, 2010, p. 587. doi:10.1088/1674-4527/10/6/008.

[14] Hasnain, Z., Lamb, C., and Ross, S. D., “Capturing near-Earth Asteroids around Earth,” Acta Astronautica, Vol. 81, No. 2, 2012, pp. 523-531. doi:10.1016/j.actaastro.2012.07.029.

[15] Verrier, P., and McInnes, C., "Low-Energy Capture of Asteroids onto Kolmogorov-Arnold-Moser Tori," Journal of Guidance, Control, and Dynamics, Vol. 38, No. 2, 2014, pp. 330-335. doi:10.2514/1.G000797.

[16] Urrutxua, H., Scheeres, D., Bombardelli, C., Gonzalo, J., and Peláez, J., “Temporarily Captured Asteroids as a Pathway to Affordable Asteroid Retrieval Missions,” Journal of Guidance, Control, and Dynamics, Vol. 38, No. 11, 2015, pp. $2132-2145$. doi:10.2514/1.G000885.

[17] Gong, S., and Li, J., “Asteroid Capture using Lunar Flyby,” Advances in Space Research, Vol. 56, No. 5, 2015 , pp. 848-858. doi:10.1016/j.asr.2015.05.020.

[18] Bao, C., Yang, H., Barsbold, B., and Baoyin, H., "Capturing near-Earth asteroids into bounded Earth orbits using gravity assist," Astrophysics and Space Science, Vol. 360, No. 2, 2015, p. 61. doi:10.1007/s10509-015-2581-3. 
[19] Massonnet, D., and Meyssignac, B., "A Captured Asteroid: Our David's Stone for Shielding Earth and Providing the Cheapest Extraterrestrial Material," Acta Astronautica, Vol. 59, No. 1, 2006, pp. 77-83. doi:10.1016/j.actaastro.2006.02.030.

[20] Sánchez, J. P., Yárnoz, D. G., Alessi, E. M., and McInnes, C., "Gravitational Capture Opportunities for Asteroid Retrieval Missions," Proceedings of the 63rd International Astronautical Congress, Naples, Italy, 2012.

[21] Yárnoz, D. G., Sánchez, J. P., and McInnes, C., "Easily Retrievable Objects among the NEO population,” Celestial Mechanics and Dynamical Astronomy, Vol. 116, No. 4, 2013, pp. 367-388. doi:10.1007/s10569-013-9495-6.

[22] Sánchez, J. P., and Yárnoz, D. G., “Asteroid Retrieval Missions enabled by Invariant Manifold Dynamics,” Acta Astronautica, Vol. 127, 2016, pp. 667-677. doi:10.1016/j.actaastro.2016.05.034.

[23] Mingotti, G., Sánchez, J. P., and McInnes, C., "Low Energy, Low-Thrust Capture of Near Earth Objects in the SunEarth and Earth-Moon Restricted Three-Body Systems," AIAA/AAS Astrodynamics Specialist Conference, 2014 , p. 4301. doi:10.1007/s10569-009-9220-7.

[24] Tan, M., McInnes, C., and Ceriotti, M., "Direct and Indirect Capture of Near-Earth Asteroids in the Earth-Moon System," Celestial Mechanics and Dynamical Astronomy, Vol. 129, No. 1, 2017, pp. 57-88. doi:10.1007/s10569-017-9764-x.

[25] Sánchez, J. P., Alessi, E. M., Yarnoz, D. G., and McInnes, C., "Earth resonant gravity assists for asteroid retrieval missions," 64th International Astronautical Congress 2013, 2013, pp. IAC-13.

[26] Sánchez, J. P., Colombo, C., and Alessi, E. M., "Semi-Analytical Perturbative Approaches to Third Body Resonant Trajectories," 66th International Astronautical Congress 2015, 2015, pp. IAC-15.

[27] Strange, N., Landau, D., Chodas, P., and Longuski, J., "Identification of Retrievable Asteroids with the Tisserand criterion," AIAA/AAS Astrodynamics Specialist Conference, 2014, p. 4458.

[28] Sánchez, J. P., and McInnes, C., "On the Ballistic Capture of Asteroids for Resource Utilization," 62nd International Astronautical Congress, Vol. 1, 2011, pp. 1-16.

[29] Zamaro, M., and Biggs, J., "Natural and artificial orbits around the Martian moon Phobos," Ph.D. thesis, University of Strathclyde, 2015.

[30] Szebehely, V., and Grebenikov, E., “Theory of Orbits - The Restricted Problem of Three Bodies.” Soviet Astronomy, Vol. 13, 1969, p. 364.

[31] Simó, C., Gómez, G., Llibre, J., Martinez, R., and Rodriguez, J., “On the Optimal Station Keeping Control of Halo Orbits,” Acta Astronautica, Vol. 15, No. 6, 1987, pp. 391-397. doi:10.1016/0094-5765(87)90175-5.

[32] Koon, W., Lo, M., Marsden, J., and Ross, S. D., "Heteroclinic Connections between Periodic Orbits and Resonance Transitions in Celestial Mechanics," Chaos: An Interdisciplinary Journal of Nonlinear Science, Vol. 10, No. 2, 2000, pp. 427-469. doi:10.1063/1.166509. 
[33] Ross, S. D., and Scheeres, D. J., "Multiple Gravity Assists, Capture, and Escape in the Restricted Three-Body Problem,” SIAM Journal on Applied Dynamical Systems, Vol. 6, No. 3, 2007, pp. 576-596. doi:10.1137/060663374.

[34] Alessi, E. M., and Sánchez, J., "Semi-Analytical Approach for Distant Encounters in the Spatial Circular Restricted Three-Body Problem," Journal of Guidance, Control, and Dynamics, Vol. 39, No. 2, 2015, pp. 351-359. doi:10.2514/1.G001237.

[35] Öpik, E. J., "Interplanetary encounters: close-range gravitational interactions," Amsterdam, Elsevier Scientific Publishing Co.(Developments in Solar System and Space Science. Volume 2), 1976.160 p., Vol. 2, 1976.

[36] Valsecchi, G. B., “Geometric conditions for quasi-collisions in Öpik's theory,” Dynamics of Extended Celestial Bodies and Rings, Springer, 2006, pp. 145-158. doi:10.1007/3-540-32455-0_6.

[37] Wilson, S. W. J., "A pseudostate theory for the approximation of three-body trajectories," Astrodynamics Conference, 1969, p. 1061. doi:10.2514/6.1970-1061.

[38] Parvathi, S. P., and Ramanan, R. V., "Iterative Pseudostate Method for Transfer Trajectory Design of Interplanetary Orbiter Missions," Journal of Guidance, Control, and Dynamics, 2016, pp. 2799-2809. doi:10.2514/1.G002072.

[39] MPC Asteroid Database, "Minor Planet Center Asteroid Database,"http://www . minorplanetcenter .net/iau/TheIndex. html 2017. Accessed: 2017-01-10.

[40] Horizons JPL Asteroid Database, “Horizons JPL Asteroid Database,”http://ssd.jpl.nasa.gov/horizons.cgi 2017. Accessed: 2017-01-10.

[41] Bombardelli, C., Gonzalo, J. L., and Roa, J., "Approximate analytical solution of the multiple revolution Lambert's targeting problem," Journal of Guidance, Control, and Dynamics, Vol. 41, No. 3, 2018, pp. 792-801. doi:10.2514/1.G002887.

[42] Herget, P., “The differential correction of orbits,” The Astronomical Journal, Vol. 48, 1939, pp. 105-108. doi:10.1086/105564.

[43] Vasile, M., and Locatelli, M., "A Hybrid Multiagent Approach for Global Trajectory Optimization," Journal of Global Optimization, Vol. 44, No. 4, 2009, pp. 461-479. doi:10.1007/s10898-008-9329-3.

[44] Patterson, M., and Rao, A., "GPOPS-II: A MATLAB software for solving multiple-phase optimal control problems using hp-adaptive Gaussian quadrature collocation methods and sparse nonlinear programming," ACM Transactions on Mathematical Software (TOMS), Vol. 41, No. 1, 2014, p. 1. doi:10.1145/2558904.

[45] Waechter, A., Laird, C., Margot, F., and Kawajir, Y., "Introduction to IPOPT: A tutorial for downloading, installing, and using IPOPT,", 2009.

[46] Patterson, M., Hager, W., and Rao, A., “A ph-mesh Refinement Method for Optimal Control,” Optimal Control Applications and Methods, Vol. 36, No. 4, 2015, pp. 398-421. doi:10.1002/oca.2114.

[47] Darby, C., Hager, W., and Rao, A., “An hp-adaptive Pseudospectral Method for Solving Optimal Control Problems,” Optimal Control Applications and Methods, Vol. 32, No. 4, 2011, pp. 476-502. doi:10.1002/oca.957. 
[48] Walker, M., Ireland, B., and Owens, J., "A set modified equinoctial orbit elements," Celestial Mechanics and Dynamical Astronomy, Vol. 36, No. 4, 1985, pp. 409-419. doi:10.1007/BF01227493. 
2018-11-26

\section{Multifidelity design of low-thrust resonant captures for near-Earth asteroids}

Neves, Rita

AIAA

Neves R, Sanchez JP. (2019) Multifidelity design of low-thrust resonant captures for near-Earth asteroids. Journal of Guidance, Control, and Dynamics, Volume 42, Issue 2, 2019, pp. 335-346 https://doi.org/10.2514/1.G003599

Downloaded from Cranfield Library Services E-Repository 